ARTICLE

\title{
Optimization of hierarchical structure and nanoscale-enabled plasmonic refraction for window electrodes in photovoltaics
}

\author{
Bing Han ${ }^{1, \star}$, Qiang Peng ${ }^{1, \star}$, Ruopeng $\mathrm{Li}^{1}$, Qikun Rong${ }^{1}$, Yang Ding ${ }^{1}$, Eser Metin Akinoglu ${ }^{2,3}$, Xueyuan Wu${ }^{4}$, \\ Xin Wang ${ }^{5}$, Xubing Lu1, Qianming Wang ${ }^{6}$, Guofu Zhou ${ }^{5}$, Jun-Ming Liu ${ }^{1,7}$, Zhifeng Ren ${ }^{8}$, Michael Giersig ${ }^{2,9}$, \\ Andrzej Herczynski ${ }^{4}$, Krzysztof Kempa ${ }^{1,4}$ \& Jinwei Gao ${ }^{1}$
}

An ideal network window electrode for photovoltaic applications should provide an optimal surface coverage, a uniform current density into and/or from a substrate, and a minimum of the overall resistance for a given shading ratio. Here we show that metallic networks with quasi-fractal structure provides a near-perfect practical realization of such an ideal electrode. We find that a leaf venation network, which possesses key characteristics of the optimal structure, indeed outperforms other networks. We further show that elements of hierarchal topology, rather than details of the branching geometry, are of primary importance in optimizing the networks, and demonstrate this experimentally on five model artificial hierarchical networks of varied levels of complexity. In addition to these structural effects, networks containing nanowires are shown to acquire transparency exceeding the geometric constraint due to the plasmonic refraction.

\footnotetext{
${ }^{1}$ Institute for Advanced Materials and Guangdong Provincial Key Laboratory of Quantum Engineering and Quantum Materials, South China Normal University, Guangzhou 510006, China. ${ }^{2}$ Department of Physics, Freie Universität Berlin, 14195 Berlin, Germany. ${ }^{3}$ Max Planck Institute of Colloids and Interfaces, 14476 Potsdam, Germany. ${ }^{4}$ Department of Physics, Boston College, Chestnut Hill, Massachusetts 02467, USA. ${ }^{5}$ Electronic Paper Displays Institute, South China Normal University, Guangzhou 510006, China. ${ }^{6}$ School of Chemistry and Environment, South China Normal University, Guangzhou 510006, China. ${ }^{7}$ Laboratory of Solid State Microstructures, Nanjing University, Nanjing 210093, China. ${ }^{8}$ Department of Physics and TcSUH, University of Houston, Houston, Texas 77204, USA. ${ }^{9}$ Helmholtz-Zentrum Berlin, Institut Nanoarchitekturen für die Energieumwandlung, 14109 Berlin, Germany. * These authors contributed equally to this work. Correspondence and requests for materials should be addressed to K.K. (email: kempa@bc.edu) or to J.G. (email: gaojw@scnu.edu.cn).
} 
igh electrical conductivity and optical transmittance of window electrodes play a crucial role in various optoelectronic devices such as solar cells ${ }^{1-3}$, lightemitting diodes (LEDs) $)^{4-6}$, displays ${ }^{7-9}$, sensors ${ }^{10-12}$ and smart windows $^{13-15}$. In photovoltaic (PV) applications (solar cells and LEDs), window electrodes must display an efficient and uniform current transport, as well as a very high light transmission into/from the semiconductor substrate ${ }^{16,17}$.

Various window electrode materials have been developed in the last century, which have been based on thin, uniform films of metal oxides, highly doped large gap semiconductors. The most common of those today is the indium tin oxide (ITO). The need for ITO replacement has recently emerged due to relatively high cost, shortage of indium, brittleness and the fact that transparency and the overall conductivity is not very good. In addition, these materials are continuous, uniform films, and thus not optimal for collection of non-uniform currents developing around contacts, and other non-uniformities in the PV structures, thus affecting their efficiency.

In this context, it has been argued that quasi-fractal (QF) or hierarchical metallic networks could be a good ITO replacement, optimal for the required function of efficient and uniform current transport, as well as a very high light transmission into/from the semiconductor substrate ${ }^{18}$. In an analogous problem of the heat flow, it was demonstrated that QF structures lead to a marked reduction of the thermal resistance ${ }^{19}$. A similar problem of nutrient delivery into a leaf's tissue, with minimal light shading, has been solved by the natural evolution with a QF hierarchical network of the leaf-venation system ${ }^{18-21}$. It is also known that river tributaries from a basin develop a self-similar structure of branching to homogenize water flow ${ }^{22}$.

In this work, we first propose a theoretical analysis of window electrode networks subject to the following optimization conditions: (i) a maximal surface coverage; (ii) a uniform current density; and (iii) a minimum of the overall resistance at fixed shading. The first condition implies a fractal structure of dimension approaching 2 . We show that the third condition is incompatible with both the first and the second. However, a QF tree with a slightly modified scaling in the vertical can, in the limit, satisfy the first and the last conditions perfectly, and also the second allowing for discontinuities of in-plane current densities. We then verify our theoretical analysis with a series of systematic and detailed experiments and simulations.

\section{Results}

Properties of QF hierarchical structures. To understand why fractal-like electrodes prove superior to other networks, consider a hypothetical ideal window electrode for PV applications. Since the efficiency of collecting current can always be increased at the cost of shading, assume fixed shading, defined as a fraction of the area of the light-harvesting surface. For such a constant shading, the theoretical ideal electrode would have the following three properties: (i) optimal coverage of the surface; (ii) uniform current density; and (iii) minimum in-plane resistance overall. Optimal coverage, whereby current is effectively collected from the entire surface, requires a progressively finer network of branches at smaller and smaller scales. If the overall network is to be near homogenous, a self-similar, surface-covering structure is required. Thus, condition (i) implies fine structure and self-similarity (fractal network) in the plane. Furthermore, near-perfect coverage of the surface implies a fractal of dimension $D$ approaching 2 . The simplest network is one with the branching number $N=2$, which is a pattern where at each order a given parent branch splits into two daughter sub-branches, a fractal tree $\mathrm{e}^{21-24}$.

Consider first, for simplicity, an electrode in the form of a fractal tree with the square cross-section (the actual geometry of the cross-section does not matter for any of the results below, although it may affect the quality of the electrical contact). At a junction of order $n$ of such a tree, a branch of width $r_{n}$ and length $l_{n}$ splits into two branches of widths and lengths $r_{n+1}$ and $l_{n+1}$, respectively (it is sufficient here to consider trees with identical daughter branches at each junction). Continuity of current through the junction, assuming fixed current density $J_{o}$, condition (ii), gives

$$
r_{n}=\sqrt{2} r_{n+1}
$$

Consequently, the linear dimensions of the branches scale with the factor $1 / s$ where $s=\sqrt{2}$, and the widths at each junction satisfy $r_{n}^{q}=2 r_{n+1}^{q}$ with $q=2$. Uniformity of current density along each branch of the junction is now assured. Furthermore, since the area of contact between a given branch and the substrate is equal to the area of contact of its two daughter branches, $r_{n} l_{n}=2 r_{n+1} l_{n+1}$, continuity of current guarantees also the uniformity of current density $J_{\mathrm{s}}$ from the substrate into the electrode. Indeed, $J_{o}=J_{\mathrm{s}} A_{\mathrm{s}} / r_{o}^{2}$, where $A_{\mathrm{s}}$ is the total shading area. The fractal dimension corresponding to this scaling is

$$
D=\frac{\ln N}{\ln s}=\frac{\ln 2}{\ln \sqrt{2}}=2
$$

Evidently, conditions (i) and (ii) favour an electrode, which is a QF (truncated) tree of the fractal dimension approaching 2, in our hypothetical, idealized scenario.

We now turn to condition (iii). It has been shown rigorously that an electrode in the form of fractal tree has optimal properties for electrochemical applications, including minimal resistance ${ }^{23}$. Here we derive the exponent $q$, which minimizes electrical resistance with the in-plane cross-sectional area (shading) kept constant. To do this, we consider resistances of the branches meeting at a junction and employ the Lagrange multiplier method. The resistance of the parent branch at order $n$ is given by $R_{n}=\rho_{o} l_{n} / r_{n}^{2}$, where $\rho_{o}$ is the resistivity of the electrode material. Assuming the ratio $l_{n} / r_{n}=b$ is a constant, $R_{n}=a r_{n}^{-1}$, where $a=\rho_{o} b$ is a constant. It follows that the resistance of the segment of the network consisting of the parent and two daughter branches (at order $n+1$ ), and the area of its cross-section, can be written as $R(x, y)=a\left(x+\frac{1}{2} y\right)$ and $A(x, y)=b\left(x^{-2}+2 y^{-2}\right)$, respectively, with $x=r_{n}^{-1}, y=r_{n+1}^{-1}$. We therefore define the functional

$$
F_{\lambda}(x, y)=a x+\frac{1}{2} a y+\lambda\left(b x^{-2}+2 b y^{-2}-A_{o}\right)
$$

and seek its minimum. Setting the derivative with respect to the Lagrange multiplier $\lambda$ to zero assures that the shading area is kept constant (and equal to $A_{o}$ ). Setting the derivatives with respect to $x$ and $y$ to zero and eliminating $\lambda$ from the resulting equations gives $x^{-3}=4 y^{-3}$ or

$$
r_{n}^{3 / 2}=2 r_{n+1}^{3 / 2}
$$

It follows that $q=D=1.5$ minimizes the net resistance, whereas $D \rightarrow 2$ is required for the uniform current density and optimal surface coverage. The two requirements cannot be satisfied simultaneously and thus a window electrode, of square or circular cross-section, which satisfies all three conditions formulated above, is impossible.

However, a branch cross-section does not need to be defined by a single parameter, and in practice it is quite unlikely to be. To take the simplest example, we assume that each branch has a rectangular cross-section, with width $r_{n}$ and height $h_{n}$. 
Furthermore, uniformity of current density is not physically necessary (as long as current is continuous as demanded by Kirchhoff's Law). By relaxing condition (ii), allowing discontinuities in current density across the junctions, and by controlling relative thickness of branches $h_{n}$ at each order, one can design a network whose projection onto the cell's plane is a fractal of the desired fractal dimension. One way to do this is to set the height of the branch at $n$-th order to scale as

$$
h_{n}=r_{0}\left(\frac{r_{n}}{r_{0}}\right)^{\alpha}=r_{0}^{1-\alpha} r_{n}^{\alpha}
$$

and require that the projection onto the plane of the cell be a (perfect) fractal of dimension $D<2$. Imposing condition (iii) for the scaling (5) now gives

$$
r_{n}^{\alpha+2}=4 r_{n+1}^{\alpha+2}
$$

that is, $D=(\alpha+2) / 2$ or $\alpha=2(D-1)$. As expected, with $D=1.5$, $\alpha=1$ and (5) reduces to $h_{n}=r_{n}$ for square electrodes. Continuity of current requires now $J_{n} r_{n} h_{n}=2 J_{n+1} r_{n+1} h_{n+1}$, which, on using (5) and (6) yields

$$
J_{n+1}=2^{\alpha /(\alpha+2)} J_{n}=2^{(D-1) / D} J_{n}
$$

For the hypothetical ideal network, $D=\alpha=2$, implying perfect coverage of the surface, height scaling given in our model by $h_{n}=r_{n}^{2} / r_{0}$, and current density increasing at each junction according to $j_{n+1}=\sqrt{2} j_{n}$. Now conditions (i) and (iii) are satisfied exactly, whereas condition (ii) assures continuity of current. A finite, real version of such a network would be optimal (save for the uniformity of current density), though of course highly cumbersome to manufacture.

It follows that our electrode based on the venation of a Magnolia alba leaf ${ }^{18}$, with the measured value $D=1.4 \pm 0.2 \approx 1.5$, is close to the best achievable under the constraint of equal width and height of branches, with small discontinuity in current density at each junction (of about $25 \%$ using our model). The only deficiency of this network is the less than maximal surface coverage.

QF network of leaf venation. To demonstrate experimentally that a fractal-like network outperforms a non-hierarchical structure, we use the QF network developed earlier by members of our team and described in detail in ref. 18. It is based on the metalized (by sputtering of silver) venation of a Magnolia alba leaf. The left inset in Fig. 1a shows an scanning electron microscopy (SEM) image of this QF network. For comparison, we chose a non-hierarchical structure of the self-assembled 'crack' (C) network ${ }^{25}$, shown in right inset in Fig. 1a, as well as the conventional ITO film. The $\mathrm{C}$ network is made by coating a substrate with a thin, sacrificial film, which micro-cracks under certain conditions, and acts as a mask during sputtering of a metal (cracking template). The lift-off of the sacrificial film produces the desired electrode network. In this work we modified our original process ${ }^{25}$ by using a much cheaper and environmentally friendly new material, egg white, as the sacrificial layer. Normally, we can roughly control the fabrication process and obtain networks with different wire width, inter-wire distance and the thickness of metal layer (Supplementary Table 1), also could fabricate large-area uniform samples (Supplementary Fig. 1). The experimental data points (transmittance $T$ versus the in-plane sheet resistance $R_{\mathrm{sq}}$ ) for our QF and C networks, and ITO film, are shown in the main part of Fig. 1a. Dashed lines in this plot represent the well-known analytical formula. ${ }^{25-27}$

$$
T=1-\frac{Z_{0}}{F R_{\mathrm{sq}}}
$$

Where $Z_{0}$ is the vacuum impedance, for two values of $F=1,200$ (blue line) and 700 (red line). $F$ is the figure of merit, often used to quantify transparent conductor performance. This formula can be easily derived by modelling a network as a uniform thin film, with a large, imaginary dielectric function, and by applying the standard Fresnel optics ${ }^{27}$. The large values of $F$ for both QF and C networks (with data points 'locked-up' between the dashed lines) indicate their very high quality as transparent conductors operating in the in-plane electron transport mode, outperforming the conventional ITO-based electrode. Figure $1 \mathrm{~b}$ shows plots of $T$ versus wavelength, indicating that the $\mathrm{QF}$ and $\mathrm{C}$ networks have a large and essentially frequency-independent $T$. Note that C networks slightly outperform QF networks in the figure of merit test, since these are optimized for the vertical, not the in-plane transport.

To test the optoelectronic performance of the films in the vertical electron transport mode (into or from a substrate), we have performed two additional experiments. In the first, we measure the contact resistance $R_{\text {cont }}$ of a film (for example, a network) to the $80 \mathrm{~nm}$ thick, flat $\mathrm{Al}$-doped zinc oxide electrode, as shown schematically in Fig. 1c. All films have been selected to have essentially identical $T$. As expected from theoretical analysis, the QF network in the vertical transport mode strongly outperforms both the C-network and ITO: $R_{\text {cont }}=2.6 \Omega(\mathrm{QF})$; $14.8 \Omega(\mathrm{C})$; and $28.4 \Omega$ (ITO). In the second experiment, we developed a series of PV cells with window electrodes based on those films. Again, all electrodes have been selected to have essentially identical $T$. Since depositing the window electrode (QF, C or ITO) on a glass substrate was the initial stage of the cell development, it allowed us to select the electrodes based on $T$. Figure 1d shows an SEM image of the cross-section of the cell (the cell processing and $I-V$ characteristic details in the Supplementary Methods). The identical processing has been used for all cells, and the completed cells have been finally selected to have identical short circuit current density and the shunt resistance, which is expected if the junctions are identical and all the window electrodes have the same $T$. The measured $I-V$ characteristics of the selected PV cells under AM1.5 illumination are shown in Fig. 1e. The inset shows that as expected, the QF window electrode provides the smallest series resistance $R_{\mathrm{s}}=23$ $\Omega, 35 \%$ smaller than the random C electrode with $R_{\mathrm{s}}=35.6 \Omega$, even though the same amount of metal was used (the same $T$ implies the same metal coverage $v$ ). Both QF and C networks have much smaller series resistances than the uniform thick film of ITO, with $R_{\mathrm{s}}=47.4 \Omega$. The energy conversion efficiencies of $5.91 \%, 5.46 \%$ and $5.37 \%$ for QF, C and ITO cells, respectively, reflect this trend also. Note that the relatively low overall efficiency of the cells is a result of missing light-trapping schemes, omitted intentionally to emphasize the electrode performance. The additional insets in Fig. 1e show photographs of the PV cells, with clearly visible leaf venation structure for the QF network cell.

Model networks. Our theoretical analysis, based on the network topology, shows that the geometrical details of the network play a secondary role. This implies that the increasing number of hierarchical orders, not network symmetry (periodic or non-periodic), should be the main consideration for a hierarchical network optimization. To test this conclusion experimentally, we developed a number of networks with varying network geometries, and a number of hierarchical orders (the schematic of the fabrication process is shown in Supplementary Fig. 2). Morphology of these networks is shown in Fig. 2. The first network (Fig. 2a) is the C network ${ }^{25,28,29}$, already discussed above. This is a first-order random network (R1). Figure $2 b$ presents an optical 

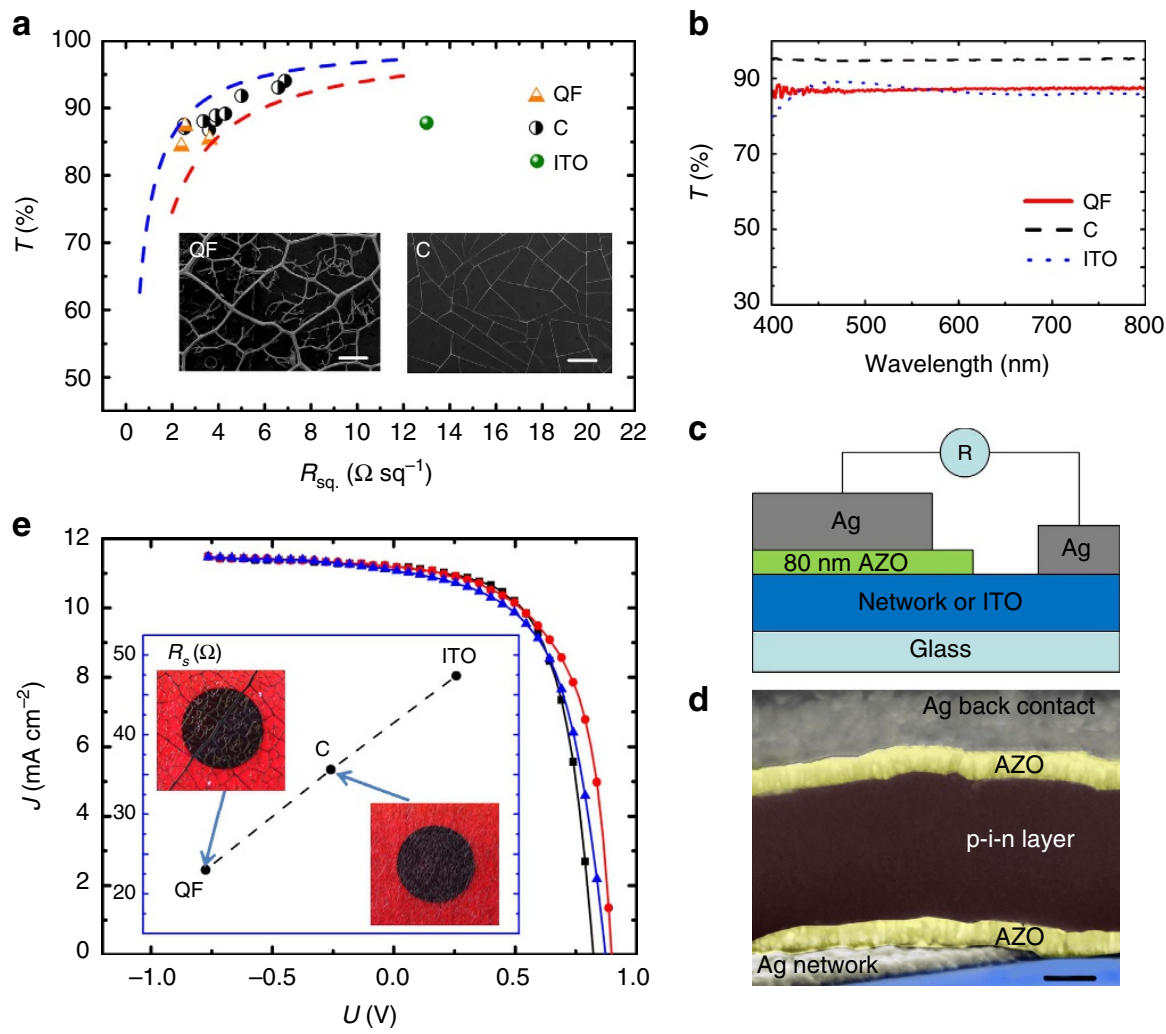

Figure 1 | Optoelectronic performance and morphology of the networks and ITO films. (a) $T$ versus $R_{\mathrm{sq}} ; T$ is measured at $550 \mathrm{~nm}$ vacuum wavelength. The expected analytical dependencies (equation (8)) for two figures of merit $F=1,200$ and 700 are shown as the blue and red dashed lines, respectively. The left inset shows the SEM image of the QF network based on the leaf venation (scale bar, $500 \mu \mathrm{m}$ ), and the right inset is the SEM image of the C network (scale bar, $100 \mu \mathrm{m}$ ). (b) T versus wavelength for QF, C and ITO films. (c) Schematic of the contact resistance measurement. (d) SEM image of the cross-section of the PV cell. The scale bar is $200 \mu \mathrm{m}$. (e) I-V characteristics of the PV cells under AM1.5 illumination: QF (red line with circles); C (black line with squares); and ITO (blue line with triangles). The inset shows a plot of the corresponding series resistance $R_{\mathrm{s}}$. Two optical images of the PV cells are also shown in this inset; the cell based on the QF network has a clearly visible leaf venation structure. The dark circle $\left(0.196 \mathrm{~cm}^{2}\right)$ in the middle of each cell is the $\mathrm{Ag}$ back reflector.

image of a large-scale simple grid network. This is a first-order periodic network (P1). Figure $2 \mathrm{c}$ is an SEM image of an ultrasmall scale grid network (UP1), developed to study plasmonic refraction, discussed later. This is a first-order ultrafine periodic network. The second-order random network (R2) is made by depositing silver nanowires (NWs) (the detail of synthesis of Ag NWs in Supplementary Methods) on the R1 network. Its SEM image (Fig. 2d) reveals the two-order hierarchy: the $\mathrm{C}$ network (first order) and the silver NW (second order). NWs have an average length of $\sim 50 \mu \mathrm{m}$ and diameter of $\sim 200 \mathrm{~nm}$. Figure $2 \mathrm{e}$ presents a high (atomic)-resolution transmission electron microscopy study of a single NW, showing an excellent quality with clearly visible, defect-free atomic planes (in lower and higher magnification), as well as almost perfect X-ray diffraction pattern. Figure $2 \mathrm{f}$ is a high-resolution SEM image of the details of R2 network, further enhanced in the inset and an atomic force microscopy (AFM) image, showing height profile of this network (Supplementary Fig. 3). Figure $2 \mathrm{~g}$ is an optical image of the second-order periodic network (P2), obtained by adding the narrow horizontal and vertical metallic lines (of $200 \mu \mathrm{m}$ length) to the P1 network. P1 and P2 large-scale networks were made by photolithography (the details are shown in the Methods section and Supplementary Fig. 2).

To start with, a study was performed of the impact of the NW density on the in-plane network performance. The results are summarized in Fig. 3a, which shows (as red dots) sheet resistance $R_{\mathrm{sq}}$ of three, C-based R2 hierarchical networks called here S1, S2 and S3, each having increased number of deposited NW. SEM images of these networks, together with the corresponding NW-free C network, are shown in Fig. 3b. Clearly $R_{\text {sq }}$ decreases strongly with increasing NW density, while the transmittance $T$ (the numbers in \% immediately above the data points) decreases much slower. This demonstrates the advantage of adding a new, lower order to a hierarchical network: while the transmittance is affected only marginally, because of a minimal shading increase due to the added fine structure, the resistance is lowered significantly due to optimization of the carrier transport via the network of 'local roads'. Further confirmation of this advantage is via PV effect. We have made PV cells using the same procedure and structure as shown in Fig. 1d. As expected, the efficiency of the R2 cell is $E_{\mathrm{f}}=6.83 \%$, much higher than the efficiency of the best corresponding PV cell based on the C network (R1) (5.89\%), and the ITO-based cell (5.21\%). For more details see Supplementary Fig. 4. As before, the relatively low absolute values of the efficiencies reflect the absence of any light-trapping scheme omitted on purpose, to emphasize the electrode performance.

To test the importance of the network symmetry, we have made four additional networks, all with the same silver thickness of $100 \mathrm{~nm}$ on glass. The first is a C-based R1 network, with random structure similar to that in Fig. 2a, and the second is the C-based corresponding R2 network. The third network is a simple square network (P1) as shown in Fig. 2f, and the fourth, the two-order periodic hierarchical (P2) network based on this square network, as shown in Fig. $2 \mathrm{~g}$. To facilitate comparisons between these four networks, they were designed to have the same 
a

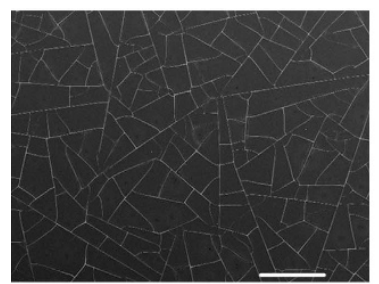

d

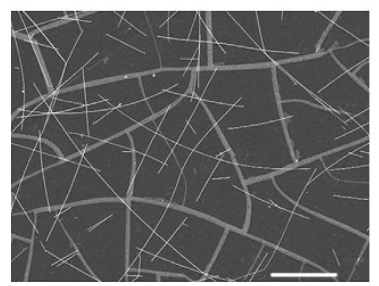

g

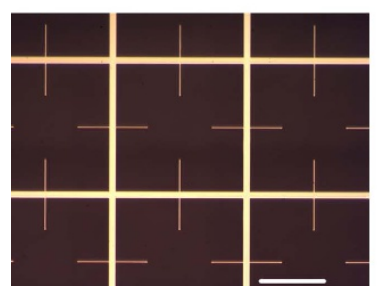

b

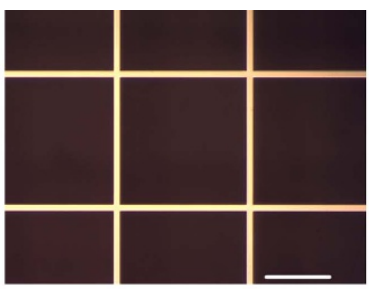

e

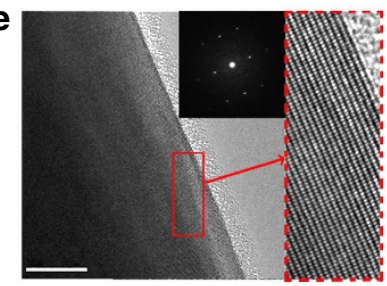

h

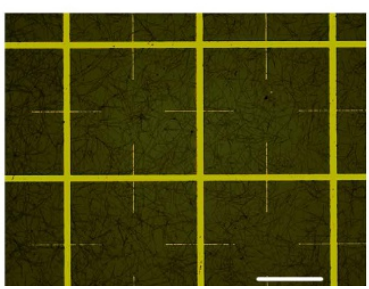

c


Figure 2 | Morphology of the model networks. Single-order networks: (a) C network (R1; SEM image; scale bar, 200 $\mu$ m); (b) large-scale simple grid network (P1; optical image; scale bar, $200 \mu \mathrm{m}$ ); and (c) ultrafine scale grid network (UP1; SEM image; scale bar, $5 \mu \mathrm{m}$ ). Two-order random networks: (d) C-based network (R2; SEM image; scale bar, $30 \mu \mathrm{m}$ ). (e) High (atomic)-resolution transmission electron microscopy images of a single NW. Inset shows an X-ray diffraction pattern. Scale bar, $10 \mathrm{~nm}$. (f) High-resolution SEM image of the R2 network at the tilt angle of $\sim 60^{\circ}$. Scale bar, $2 \mu \mathrm{m}$. Inset: details of the ribbon-NW contact (scale bar, $400 \mathrm{~nm}$ ). (g) Two-order periodic network (P2; optical image; scale bar, $200 \mu \mathrm{m})$. (h) Three-order periodic network (M3) obtained by adding NW to the P2 network. Scale bar, $200 \mu \mathrm{m}$. (i) Optical image of the quasi fractal QF network. Scale bar, $500 \mu \mathrm{m}$.

transmittance $T \sim 90 \%$ (to within $2 \%$ ), at the $550 \mathrm{~nm}$ vacuum wavelength. The contact resistance $R_{\text {cont }}$ has been measured using the set-up of Figs $1 \mathrm{c}$, and $3 \mathrm{c}$ shows $R_{\text {cont }}$ versus network order, where the blue solid circles represent random and the red solid triangles periodic networks. As expected, the contact resistance of the periodic and the random networks of the same order, and having the same $T$ is very similar. On the other hand, adding an order significantly reduces $R_{\text {cont }}$. Note that while $R_{\text {cont }}$ is very different for P1 and P2 networks, both networks must have exactly identical in-plane sheet resistances $R_{\mathrm{sq}}$, since the lower-order features of the P2 network form 'dead-end' local 'streets', which do not contribute to the in-plane transport. In addition to the networks discussed above, we also made a threeorder hierarchical mixed network (M3) by adding NW to the P2 network. Optical image of this three-order mixed (periodicrandom) network (M3) is shown in Fig. 2h. The corresponding contact resistance of this network is $R_{\text {cont }} \sim 3.7 \Omega$ (red circle in Fig. 3c), less than other networks, except for the QF leaf venation network $(\sim 2.6 \Omega)$, shown in Fig. 2i. This confirms again the importance of increasing orders of hierarchical networks.

Plasmonic effects. The networks utilizing NW have an additional benefit of the subwavelength scale of the NW in the visible frequency range. This provides conditions for plasmonic refraction effects 16,30 , which enhance the transparency above the classic super-wavelength geometric limit ${ }^{17} T_{\mathrm{g}}=1-v$, where $T_{\mathrm{g}}$ is the transmittance and $v$ is the surface fraction covered by metal. To demonstrate this, we calculated $v$ from the SEM images of the C-based R2 networks. While for the $\mathrm{C}$ network we confirmed that measured $T \approx T_{\mathrm{g}}$, the statistically averaged $T_{\mathrm{g}}$ values for S1, S2 and S3 networks are $85.7 \%, 83.4 \%$ and $78.7 \%$, respectively, all significantly smaller than the corresponding optically measured $T$ values $90.2,88.8$ and $87.5 \%$. To demonstrate that this enhancement is due to the plasmonic refraction, we use the ultrafine grid network (UP1), shown in Fig. 2c. The corresponding measured and simulated $T$ versus wavelength for this network are shown in Fig. 3d. Even though these agree only qualitatively, both are substantially greater than the corresponding $T_{\mathrm{g}}=69 \%$, except for the immediate vicinity of $\lambda=500 \mathrm{~nm}$, where the localized Mie-like plasmon is excited ${ }^{31}$. To visualize the plasmonic refraction, we also simulated electromagnetic fields in the vicinity of a single $\mathrm{NW}$ at $\lambda=950 \mathrm{~nm}$, that is, in the high- $T$ region. The simulated snapshots of the $\mathrm{x}$-component of the electric field (colour encoded) for various times $t$ are shown in the inset in Fig. 3d. The wave-front evolves from the essentially plane wave at $t=0$, to the complete partition of the wave-front at $t=T / 4$, back to almost a complete recovery of the plane wave propagation past the NW for $t=T / 2$. Clearly, only a very small back reflection occurs, as indicated by a tiny distortion of the wave-front at $t=0$, with the electromagnetic wave avoiding, and propagating around the NW. Note that the field inside the wire is zero, at all times, confirming the classic nature of the wave-metal interaction; retarded surface plasmons follow dispersion very close to the light line, and in fact transition into it for vanishing frequency. Wave propagation around the minimum due to localized plasmons (at $\lambda=500 \mathrm{~nm}$ ) is completely different, with standing waves forming, and the wave energy propagating through the NW.

\section{Discussion}

The theoretical analysis presented here starts with three postulates for a hypothetically ideal window electrode: an optimal surface coverage; a uniform in-plane current density; and minimal overall resistance (at given shading). The first two of 
b
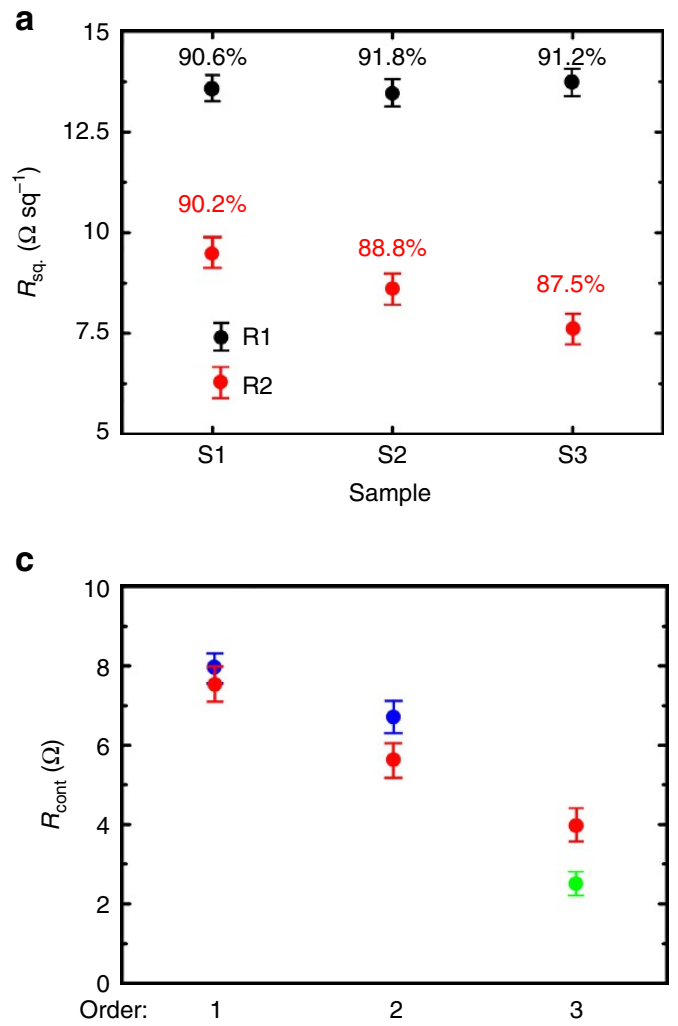

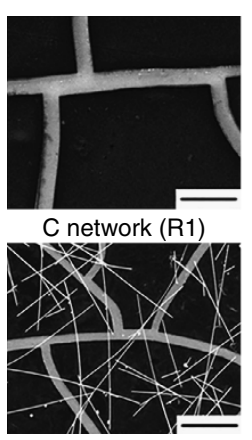

S2

d

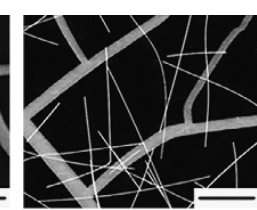

S1

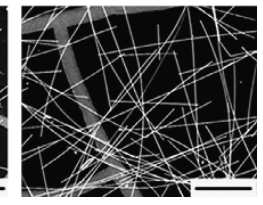

S3

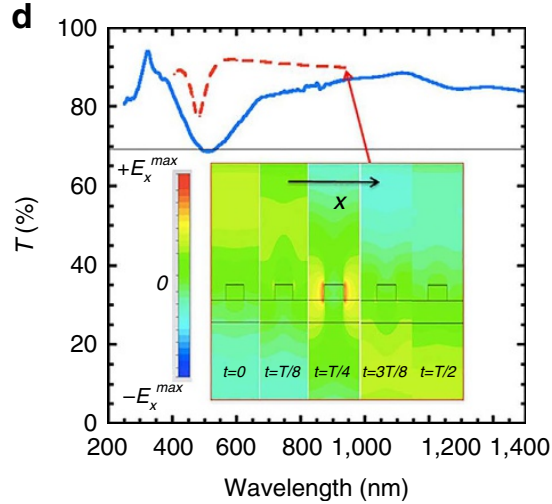

Figure 3 | Optoelectronic properties and morphology of the hierarchical networks. (a) Measured $R_{\mathrm{sq}}$ and $T$ of the C-based networks (black solid squares) and the corresponding R2 networks (red solid circles). The numbers at the data points are the corresponding $T$ values in \%. (b) SEM images of the C-based networks, with increasing NW density (C network: no NWs; S1: $0.125 \mathrm{mg} \mathrm{ml}^{-1}$; S2: $0.25 \mathrm{mg} \mathrm{ml}^{-1}$; and S3: $0.5 \mathrm{mg} \mathrm{ml}^{-1}$ ). The scale bars are all $10 \mu \mathrm{m}$. (c) Measured $R_{\text {cont }}$ for hierarchical structures of various orders (each pair of symbols represents the same order). Blue circles represent random (R1 and R2) and red triangles periodic (P1 and P2) networks. The third-order networks are M3 (red circle) and QF (green square). (d) Transmittance of the ultrafine scale grid network (UP1) shown in Fig. 2c: experimental (solid blue line); simulated (dashed red line); and $T_{g}$ (solid black). The inset shows maps of the $x$-component of the electric field near a single nanowire of the ultrafine network at various times, within the period $T$. The uncertainty in (a) and (c) comes from the non-uniformity of the film, and is calculated as a standard deviation.

these conditions require a fractal structure of dimension approaching 2, which of course is an idealization. The third condition, implying $D=1.5$, while manifestly incompatible with the first two, suggests that an electrode consisting of a truncated fractal structure of this dimension would be optimal except for less than perfect surface coverage. This explains why a network based on the veins of Magnolia alba leaf, with $D \sim 1.4$, proves to be exceptional-at least within the class of quasi fractal networks in three dimensions.

Indeed, in a series of detailed experiments, we showed that our 'natural' electrode had a significantly higher PV efficiency and smaller contact resistance, than any uniform, non-hierarchical random network, as well as the conventional ITO layer, confirming our conclusions.

These results notwithstanding, the requirement of uniform current density is not really necessary, and in fact usually not satisfied in artificial networks. Furthermore, it is desirable only insofar as to assure uniform current density into and/or from the substrate, itself not critical. Mathematically, uniformity of current density means that the network must be a fractal tree in three dimensions. We therefore designed a network, which is not fractal as a whole, but whose projection onto the window's plane is fractal-and of any desired dimension $D$, up to and including 2 . Continuity of current through every junction now determines the necessary jumps of current density. It follows that a truncated version of such a network, set to $D=2$, would theoretically be optimal, though it may be impractical to manufacture.
Beyond this theoretical result, our analysis indicates that a hierarchal structure (with multiple branching orders), rather than detailed geometry, is critical for optimizing network electrodes. We demonstrated this experimentally in a series of systematic electro-optical and PV experiments, in five hierarchical networks: a one- and two-order random, one- and two-order periodic and a three-order mixed structures. Our experimental results are summarized in Fig. 3. Figure 3d, in particular, demonstrates this conclusion explicitly. At fixed transmittance, changing from random to periodic structures, while keeping the number of hierarchical orders fixed, lowers the contact resistance only slightly, but adding another hierarchical order, for both period and random structures, reduces it by $30-50 \%$. All the while, the in-plane resistance remains essentially unchanged.

In addition to these structural effects, controlled by network hierarchy with topological elements (branching), the networks based on NWs achieve transparency exceeding the geometric limit. This is due to plasmonic refraction, which involves propagating surface plasmons transporting energy around NWs, effectively 'squeezing' light into the spaces between NW, and thus minimizing reflection. We demonstrate this effect on our ultrafine grid network, whose transparency has been measured and simulated. To visualize the plasmonic refraction, we performed also simulations of the electromagnetic fields of waves propagating through the network at various times, and for varied wavelength. Our simulations show that an electromagnetic wave couples to the surface plasmon wave in the metal, which in turn 
transports its energy seamlessly around the NW. The net result is an increased transmission of the wave through the network.

Our low-cost, self-assembled networks are mechanically very stable and flexible, surviving numerous bending cycles (for an example see Supplementary Fig. 5 and Supplementary Methods). For comparison, the resistance of ITO film changes by two orders of magnitude already after a few cycles. Such desirable mechanical stability and flexibility, combined with excellent electro-optical parameters demonstrated here, make these networks very attractive. They are suitable, in particular, for solar cells, LED lighting and transparent flexible heaters (Supplementary Fig. 6). Our study opens a path towards a new generation of smart TC, through hierarchical multi-order networks with the lowest orders at nanoscopic scales, and possibly also through intrinsic TC functionalities, such as light trapping and/or nonlinear light processing. These smart TC could eventually lead to a new class of applications such as the energy recovery, temperature and haze controlling electro-chromic windows, and high-power light sources, to name a few.

Finally, we note that while our most efficient electrode to date is based on a natural biological network, the mathematical construct we developed suggests that a superior electrode can yet be envisioned, though it seems impractical at this stage.

\section{Methods}

Fabrication of random networks R1 and R2. A pure egg white was diluted with deionized water to the concentration of about $0.6 \mathrm{~g} \mathrm{ml}^{-1}$. Spin coating (Spin coater, Laurell, USA) was used to deposit as-prepared egg white film on substrates. These were dried in air (temperature range $25-70^{\circ} \mathrm{C}$ ). After several minutes, the selfinduced cracking process occurs. A set of samples, with averaged crack width of $0.5-5 \mu \mathrm{m}$ and averaged size of the gel island of $20-500 \mu \mathrm{m}$, were obtained by varying the spin-coating speed (200-800 r.p.m.), coating time (10-50 s), concentration of sol-gel and the drying temperature. The cracking template morphology can be controlled with these fabrication parameters (details in Supplementary Table 1). The sputtering system (AJA International, ATC Orion 8, USA) was used to deposit Ag films, with thickness controlled in the range of $\sim 100 \mathrm{~nm}$. Rinsing with deionized water for 1-2 min was used to remove the sacrificial layer of the egg white film. This way we made all the R1 C-based networks. The R2 networks were manufactured by coating R1 networks with different concentration of Ag NWs. The schematic of fabrication processes (R1 and R2) is shown in Supplementary Fig. 2a).

Fabrication of hierarchical networks. Spin coating (Spin coater, Laurell, USA) was used to deposit as-prepared U-8 photoresist film on substrates. Next, the film was pre-baked in $90^{\circ} \mathrm{C}$, and then exposed to ultraviolet light for about $10 \mathrm{~s}$ through two different masks: one for the P1 and the other for the P2 network (details in Supplementary Fig. 2b,c). Finally, the sample was developed in the developing liquid for about $45 \mathrm{~s}$. The sputtering system (AJA International, ATC Orion 8, USA) was used to deposit Ag films. The thickness of Ag film was about $100 \mathrm{~nm}$. Rinsing with anhydrous alcohol for 1-2 min was used to remove the sacrificial layer of the photoresist film. The third-order M3 network was obtained by coating the P2 network with Ag NWs (details in the Supplementary Fig. 2d). UP1 structure was fabricated by using a modified nanosphere lithography, as described in detail in ref. 31. This technique, which produces an array of parallel lines was used twice, in combination with the sample rotation, to yield the final structure.

Morphology. Morphologies of samples were characterized in a SEM system (JEOL JCM-5700, Tokyo, Japan), and by using an optical microscope (MA 2002, Chongqing Optical \& Electrical Instrument Co.). High (atomic)-resolution transmission electron microscopy images were obtained using the transmission electron microscope (JEOL JEM-2100HR, Japan) operating at an acceleration voltage of $200 \mathrm{kV}$. AFM image and height profile was obtained with the AFM (Cypher, Asylum Research, USA) in a.c. mode.

Transmittance measurements. Optical transmittance was measured in an integrating sphere system (Ocean Optics, USA). Transmittance measurements have been normalized to the absolute transmittance through the substrate Polybutylene terephthalate (PET) or glass.

Electrical conductivity measurements. The sheet resistance $R_{\mathrm{sq}}$ measurements were obtained by using the van der Pauw method, as described in ref. 25 . The contact resistance $R_{\text {cont }}$ measurements were made by coating a half of a network sample with $80 \mathrm{~nm}$ of the Al-doped zinc oxide film, and then applying a fixed structure of contacts, as schematically shown in Fig. 1c.

Simulations. The simulations of the transmittance and the field maps for the network UP1 used the finite difference time domain and finite difference frequency domain code CST (https://www.cst.com/products/CSTMWS).

Data availability. The authors declare that all data supporting the findings of this study are available within the article and its Supplementary Information files.

\section{References}

1. Kim, A., Won, Y., Woo, K., Kim, C.-H. \& Moon, J. Highly transparent low resistance $\mathrm{ZnO} / \mathrm{Ag}$ nanowire/ $\mathrm{ZnO}$ composite electrode for thin film solar cells. ACS Nano 7, 1081-1091 (2013).

2. Guo, C. F. \& Ren, Z. Flexible transparent conductors based on metal nanowire networks. Mater. Today 18, 143-154 (2015).

3. Gao, J. et al. Transparent nanowire network electrode for textured semiconductors. Small 9, 733-737 (2013).

4. Yu, Z. et al. Highly flexible silver nanowire electrodes for shape-memory polymer light-emitting diodes. Adv. Mater. 23, 664-668 (2011)

5. Liang, J., Li, L., Niu, X., Yu, Z. \& Pei, Q. Elastomeric polymer light-emitting devices and displays. Nat. Photon. 7, 817-824 (2013).

6. Shin, S., Yang, M., Guo, L. J. \& Youn, H. Roll-to-roll cohesive, coated, flexible, high-efficiency polymer light-emitting diodes utilizing ITO-free polymer anodes. Small 9, 4036-4044 (2013).

7. Lee, J. et al. Very long Ag nanowire synthesis and its application in a highly transparent, conductive and flexible metal electrode touch panel. Nanoscale 4, 6408-6414 (2012).

8. Vuorinen, T. et al. Printable, transparent, and flexible touch panels working in sunlight and moist environments. Adv. Funct. Mater. 24, 6340-6347 (2014).

9. Han, S. et al. Fast plasmonic laser nanowelding for a $\mathrm{Cu}$-nanowire percolation network for flexible transparent conductors and stretchable electronics. Adv. Mater. 26, 5808-5814 (2014).

10. Harada, S., Honda, W., Arie, T., Akita, S. \& Takei, K. Fully printed, highly sensitive multifunctional artificial electronic whisker arrays integrated with strain and temperature sensors. ACS Nano 8, 3921-3927 (2014).

11. Gong, S. et al. A wearable and highly sensitive pressure sensor with ultrathin gold nanowires. Nat. Commun. 5, 3132 (2014).

12. Pang, C. et al. A flexible and highly sensitive strain-gauge sensor using reversible interlocking of nanofibres. Nat. Mater. 11, 795-801 (2012).

13. Dyer, A. L. et al. A vertically integrated solar-powered electrochromic window for energy efficient buildings. Adv. Mater. 26, 4895-4900 (2014).

14. Qiu, T. et al. Hydrogen reduced graphene oxide/metal grid hybrid film: towards high performance transparent conductive electrode for flexible electrochromic devices. Carbon 81, 232-238 (2015).

15. Baetens, R., Jelle, B. P. \& Gustavsen, A. Properties, requirements and possibilities of smart windows for dynamic daylight and solar energy control in buildings: a state-of-the-art review. Sol. Energy Mater. Sol. Cells 94, 87-105 (2010).

16. Sun, T. et al. A broadband solar absorber with $12 \mathrm{~nm}$ thick ultrathin a-Si layer by using random metallic nanomeshes. Appl. Phy. Lett. 104, 251119 (2014).

17. Sun, T. et al. Enhanced broad-band extraordinary optical transmission through subwavelength perforated metallic films on strongly polarizable substrates. Appl. Phys. Lett. 102, 101114 (2013).

18. Han, B. et al. Bio-inspired networks for optoelectronic applications. Nat. Commun. 5, 5674 (2014).

19. Neagu, M. \& Bejan, A. Constructal-theory tree networks of 'constant' thermal resistance. J. Appl. Phys. 86, 1136 (1999).

20. Zhou, H., Fan, T. \& Zhang, D. Biotemplated materials for sustainable energy and environment: current status and challenges. ChemSusChem 4, 1344-1387 (2011).

21. Tan, J. \& Saltzman, W. M. Biomaterials with hierarchically defined micro- and nanoscale structure. Biomaterials 25, 3593-3601 (2004).

22. Campos, D., Fort, J. \& Méndez, V. Transport on fractal river networks: application to migration fronts. Theor. Popul. Biol. 69, 88-93 (2006).

23. Park, B. Y., Zaouk, R., Wang, C. \& Madou, M. J. A case for fractal electrodes in electrochemical applications. J. Electrochem. Soc. 154, P1-P5 (2007)

24. Mandelbrot, B. B. The Fractal Geometry of Nature/Revised and Enlarged Edition (W.h.Freeman, 1983)

25. Han, B. et al. Uniform self-forming metallic network as a high-performance transparent conductive electrode. Adv. Mater. 26, 873-877 (2014).

26. Ellmer, K. Past achievements and future challenges in the development of optically transparent electrodes. Nat. Photon. 6, 809-817 (2012).

27. Hecht, D. S., Hu, L. \& Irvin, G. Emerging transparent electrodes based on thin films of carbon nanotubes, graphene, and metallic nanostructures. Adv. Mater. 23, 1482-1513 (2011). 
28. Gupta, R., Rao, K. \& Kulkarni, G. U. Transparent and flexible capacitor fabricated using a metal wire network as a transparent conducting electrode. RSC Adv. 4, 31108-31112 (2014).

29. Rao, K., Hunger, C., Gupta, R., Kulkarni, G. U. \& Thelakkat, M. A cracked polymer templated metal network as a transparent conducting electrode for ITO-free organic solar cells. Phys. Chem. Chem. Phys. 16, 15107-15110 (2014).

30. Gao, T. \& Leu, P. W. The role of propagating modes in silver nanowire arrays for transparent electrodes. Opt. Express 21, A419-A429 (2013).

31. Patoka, P., Sun, T., Giersig, M., Ren, Z. F. \& Kempa, K. Nanoribbon plasmonic gratings and their anomalous interaction with electromagnetic waves. $A d v$. Mater. 24, 3042-3045 (2012).

\section{Acknowledgements}

This work is supported by NSFC grant (No. 51571094), the National Key Research Programme of China (2016YFA0201002), Guangdong province grants (2014B090915005, 2014A030313447, 2013KJCX0056 and HD14CXY010). K.K. thanks the Boston College Ignite Program for additional financial support. J.M. thanks the NSFC grant (No. 51431006). This work has been partially supported from Program for Changjiang Scholars and Innovative Research Team in University (No. IRT13064), Guangdong Innovative Research Team Program (No. 2011D039), 'China National Undergraduate Innovation Experiment Program' and 'SCNU Golden seed platform'.

\section{Author contributions}

J.G. and B.H. developed the idea; B.H., Q.P. and R.L. prepared the samples and performed most of the experiments; K.K. guided the work and performed theoretical analysis of the transport and electromagnetic properties, including plasmonic refraction; A.H. performed optimization study of the QF networks; E.M.A. developed the UP1 plasmonic structure and performed its measurements; X.W. performed simulations of the UP1 structure; M.G. performed the microscopic studies of NW; J.G., K.K. and A.H. wrote the paper. All authors discussed the results and commented on the manuscript. J.G. directed the research.

\section{Additional information}

Supplementary Information accompanies this paper at http://www.nature.com/ naturecommunications

Competing financial interests: The authors declare no competing financial interests.

Reprints and permission information is available online at http://npg.nature.com/ reprintsandpermissions/

How to cite this article: Han, B. et al. Optimization of hierarchical structure and nanoscale-enabled plasmonic refraction for window electrodes in photovoltaics. Nat. Commun. 7:12825 doi: 10.1038/ncomms12825 (2016).

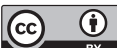

This work is licensed under a Creative Commons Attribution 4.0 International License. The images or other third party material in this article are included in the article's Creative Commons license, unless indicated otherwise in the credit line; if the material is not included under the Creative Commons license, users will need to obtain permission from the license holder to reproduce the material. To view a copy of this license, visit http://creativecommons.org/licenses/by/4.0/

(C) The Author(s) 2016 